\title{
THE ANALYSIS OF TRANSFORMER'S CONDITION BASED ON BAYES- DISCRIMINANT METHOD WITH FAULT-TREE FUZZY EVALUATION
}

\author{
Dianyang LI ${ }^{1,2}$, Shanyuan WANG ${ }^{1}$, Yujie ZHANG ${ }^{1}$, Jian FENG ${ }^{1}$, \\ Hongzhe WANG ${ }^{2}$, Ling QIN ${ }^{2}$ \\ ${ }^{1}$ Northeastern University,College Of Information Science and Engineering, 110004 \\ ${ }^{2}$ State Grid liaoning Electric Power Co, 110004.2841385779@qq.com
}

\begin{abstract}
China's electric power construction is renewing Increasingly, and the network is complex and changeable where the automation is getting higher. In this paper, Fuzzy evaluation system is established according to fault tree, and the estimation of transformer's state is judged by analytic hierarchy process. Bayesdiscriminant and discriminant formula are used to discriminate transformer's attributes, which are based on historical data. The machine identification of transformer faults combines the fuzzy evaluation and Bayesdiscriminant. It's accuracy can be improved by correcting parameters. This method can effectively avoid subjective interference caused by artificial weights. The example shows that this method could be applied to judge health status of electric power equipment and this method can play an early-warning role in the operation of monitoring system.
\end{abstract}

Keywords: Oil-immersed power transformer; fault tree analysis; Fuzzy-evaluation system; Bayes-discriminant; Health -operation

\section{INTRODUCTION}

China's State Grid Corporation revised the technical standards for power grid equipment maintenance and repair, collected and compiled a number of technical standards since 2008, which included the technical standards for maintenance of $\mathrm{AC}$ transmission and transformation equipment, high voltage DC power state maintenance technical standards, technical standards for condition maintenance of distribution network equipment and monitoring technology of power equipment condition. These standards provide a reference guarantee for the company's system to fully implement state maintenance. This paper focuses on the overhaul research of $35 \mathrm{kV}$ oil-immersed transformer, and establishes a fuzzy evaluation system based on fault tree analysis, and uses Bayesian discriminant method to improve it's accuracy.

Traditional evaluation methods such as Fuzzy Comprehensive Evaluation rely on a large amount of historical experience and subjective factors ${ }^{[1]}$. This paper improved the main defects of the Bayesian method. The Bayesian discriminant method and the fuzzy evaluation method are used to test the results. The results are compared with historical data target, then the revised opinions and methods are proposed.

Compared with the fuzzy evaluation method, Bayesian discrimination relies on fewer artificial weight factors, which reduces the subjectivity of the emergency evaluation results. Secondly, the expert database plays a role in the rapid assessment of the dispatcher's decision-making, and replaces the traditional evaluation with more objective machine discrimination, which is of great significance in the power grid disaster early warning decision.

The research on power equipment and fault assessment methods at home and abroad has grown vigorously in the past 20 years, and has formed a scientific and rigorous theoretical basis. Literature [2] summarizes the shortcomings of GAMIT/GLOBK, normalized root mean square, and proposes to manually correct the calculation error, otherwise the calculation result is too large. The author selects five parameters as a discriminating factor, and establishes a Bayesian prediction classification model for the solution. The experimental results show that the Bayesian discriminant model has good predictability. The literature [3] can not describe the dynamic logic relationship problem for the traditional fault tree, and proposes Dynamic logic such as functionrelated gates and the priority gate method. It provides ideas for the establishment of power transformer fault trees. The literature [4-5] explains the different sources of analysis information for equipment fault diagnosis, and how the collected data can be used to determine the state of the equipment. The processing is analyzed and has reference significance for power transformer fault diagnosis. Support vector machine ${ }^{[6]}$, Fuzzy matrix [7], the attribute selection algorithm and KNN network ${ }^{[8]}$ are also used for fault diagnosis. 
In this paper, the fault states of each component are classified, and the different states of each index are weighted to form a risk weight assessment. At the same time, the synergy of Bayesian network discriminant and fuzzy evaluation methods has important guiding significance for predicting the health status of transformers.

\section{OIL-IMMERSED TRANSFORMER CHARACTERIZATION ACQUISITION AND EVALUATION METHOD}

\subsection{Related information volume}

The characterization of transformer status assessment is divided into oil chromatographic index, operation of electrical test accessories, and test results of insulating oil. By measuring the dissolved gas chromatogram of the transformer, you can understand the degree of insulation degradation and forecast the remaining life of the transformer. The actual measurement shows that this classic detection method can help the maintenance workers to check the obvious faults inside the transformer and the danger of latent.

The oil acts as insulation and heat dissipation inside the large transformer. When equipment components are aging or damaged, the oil will produce different contents of low molecular hydrocarbons and impurity gases such as hydrogen. The main dissolved gas is the composition of nitrogen and oxygen. By the change in the content of the inclusion gas generated by the oil, we can determine the health of the transformer.

A way of judging the inferiority of the transformer oil and gas and indirectly judging whether the transformer is aging is to calculate the absolute gas production rate and the relative gas production rate in the transformer. The calculation formula is as follows:

$$
\begin{gathered}
v_{0}=\left(c_{1}-c_{0}\right) y /(\Delta t * s) \\
v_{1}=\left(c_{1}-c_{0}\right) /\left(c_{0} \Delta t\right) \times 100 \%
\end{gathered}
$$

In the formula, the symbol of acetylene is $c_{0}$, and the symbol of total hydrocarbon is $c_{1}$, $\Delta t$ indicate the sampling process time, $y$ indicate the total internal oil amount of the transformer, $s$ indicate the oil density, the absolute gas production rate is $v_{0}$, and the relative gas production rate is $v_{1}$. The maximum value of the rate is shown in Table 1.

Table 1. Production rate of gas

\begin{tabular}{lcc}
\hline Gas & Relative & absolute \\
\hline TH & 0.6 & 36 \\
CO & 0.5 & 30 \\
\hline
\end{tabular}

The max content of several gases are shown in Table 2, they cannot exceed the specified value.
Table 2.Max content of dissolved gases in power transformer

\begin{tabular}{ccc}
\hline \multirow{2}{*}{ Gas } & \multicolumn{2}{c}{ Content/ $\left(u L \cdot L^{-1}\right)$} \\
\cline { 2 - 3 } & $220 \mathrm{kV}$ & $330 \mathrm{kV}$ \\
\hline $\mathrm{TH}$ & 150 & 150 \\
Acetylene & 5 & 1 \\
Oxygen & 150 & 150 \\
\hline
\end{tabular}

The evaluation of the electrical test focuses on the detection of factors such as the insulation resistance of the core, the insulation resistance of the winding, and the loss factor of the insulation of the winding. The cooling device in the operating state of the accessory directly affects the operating temperature of the transformer, and the temperature indirectly affects the performance and life of the device. Therefore, the state of the casing, the cooling device, the pressure release device and the protection device need to be evaluated. In Table 3 ,the phase difference of Core inulation resistance should not be greater than $1 \%$ of the three-phase average, and the loss coefficient of other indexs is not more than $0.8 \%$.

\begin{tabular}{|c|c|c|}
\hline Index & Reason & Claim \\
\hline $\begin{array}{l}\text { Core inulation } \\
\text { resistance }\end{array}$ & $\begin{array}{l}\text { Examine the conection } \\
\text { of winding insulation } \\
\text { and current }\end{array}$ & $<1 \%$ \\
\hline $\begin{array}{l}\text { Winding insulation } \\
\text { resistance }\end{array}$ & $\begin{array}{l}\text { n Judge the performance } \\
\text { of winding insulation }\end{array}$ & \\
\hline $\begin{array}{l}\text { Winding insulation } \\
\text { dielectric loss }\end{array}$ & $\begin{array}{l}\text { Judge the defects } \\
\text { in transformers }\end{array}$ & \\
\hline
\end{tabular}

Table 3.Indexes of electrical test

In Table 4, the status of an accessory depends on its physical properties and chemical structure.

\begin{tabular}{|c|c|c|}
\hline \multirow{2}{*}{ index } & \multicolumn{2}{|c|}{ Operating status } \\
\hline & Score: $0 \sim 0.5$ & Score:0.5 1 \\
\hline Casing & Deviation & Accurate \\
\hline Cooling device & $\begin{array}{l}\text { Severe noise, } \\
\text { more dirt }\end{array}$ & $\begin{array}{l}\text { Lower noise, } \\
\text { less dirt }\end{array}$ \\
\hline $\begin{array}{l}\text { Pressure release } \\
\text { device }\end{array}$ & Severe aging & Good contact \\
\hline Protective device & Poor seal & Well seal \\
\hline
\end{tabular}

Table 4. Attachment operation

The insulating oil ensures the normal operation of the internal components of the transformer. If the insulating oil is oxidized during the process of contact with air, it will endanger the safe and stable operation of the power transformer. Table 5 shows every content's max threshold, for volume resistivity, dielectric loss coefficient is not greater than 0.04

This article uses a 1-point scoring method to evaluate various indicators of power transformers. The status division and early warning of power transformers are shown in Table 6 


\begin{tabular}{ccc} 
& \multicolumn{2}{c}{ Table 5. Indexes of insulating oil test } \\
\hline Index & \multicolumn{1}{c}{ Reason } & Claim \\
\hline $\begin{array}{c}\text { Oil medium } \\
\text { content }\end{array}$ & $\begin{array}{l}\text { Tested the aging of } \\
\text { solid insulation materials }\end{array}$ & $<2.5 \mathrm{mg} / \mathrm{L}$ \\
$\begin{array}{c}\text { Volume } \\
\text { resistivity }\end{array}$ & $\begin{array}{l}\text { Test the aging degree of the } \\
\text { transformer under the action of } \\
\text { the electric field }\end{array}$ & $<0.04$ \\
Interface & $\begin{array}{l}\text { A measure of the withstand } \\
\text { poltage capability of insulating } \\
\text { oil }\end{array}$ \\
\hline
\end{tabular}

Table 6. Statues and warnings of power transformer

\begin{tabular}{llcl}
\hline Score & Statues & Method & cycle \\
\hline $0 \sim 0.25$ & Normal & Uninterrupted & Normal \\
$0.25 \sim 0.5$ & Attention & Routine & Overdue \\
$0.5 \sim 0.75$ & Abormal & Local & Appropriate \\
$0.75 \sim 1$ & Serious & Overall & Immediately \\
\hline
\end{tabular}

Half-ladder model includes Half-lift ladder model and Half-step ladder model. When the value is larger, the better, the state quantity adopts the Halflift ladder model, otherwise the Half-step ladder model is used. In order to facilitate subsequent calculations, the quantized data is subjected to isotropic processing, that is, the quantized results are all located in the interval $[0,1]$, and the smaller the value, the better the corresponding state and running status.

The expression of the Half-lift ladder scoring model is:

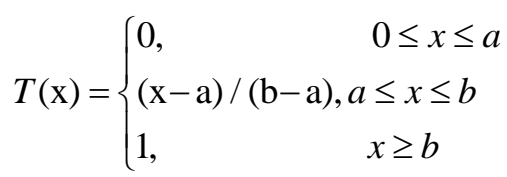

The expression of the Half-step ladder scoring model is:

$$
T(\mathrm{x})= \begin{cases}1, & 0 \leq x \leq a \\ (\mathrm{~b}-x) /(\mathrm{b}-\mathrm{a}), & a \leq x \leq b \\ 0, & x \geq b\end{cases}
$$

Among them, a, b are thresholds; $\mathrm{m}$ is a quantized parameter value. According to the relevant regulations and maintenance experience settings, the revision of the threshold and parameter values of each state quantity can be found in section 2.1.

\subsection{Establishing a characterization system}

This paper constructs a three-level system including target indicators, first-level indicators and second-level indicators. The operating status of $35 \mathrm{kV}$ oil-immersed transformers is taken as the target index. The corresponding first-level indicators can be divided into oil chromatographic analysis, electrical test, accessory operation and insulating oil test indicators. The four first-level indicators correspond to 15 secondary indicators, which are acetylene content, hydrogen content, total hydrocarbon relative gas production rate, total hydrocarbon absolute gas production rate, carbon monoxide absolute gas production rate, core insulation resistance, winding insulation resistance, Winding insulation dielectric loss, bushing, cooling device, pressure relief device, protection device, oil medium content, volume resistivity and interface gravity.

Thus, the oil immersed transformer operating state can construct a state evaluation system as shown in FIG1.

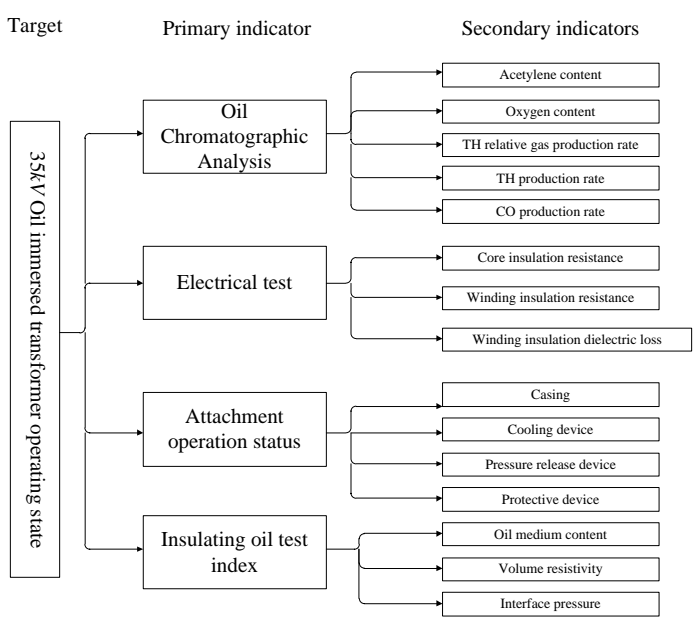

Fig. 1. The state-evaluation system of $35 \mathrm{kV}$ transformer

\section{CHARACTERIZATION OF OIL- IMMERSED TRANSFORMERS}

\subsection{Fault Tree Analysis of Characterization Information}

The fault tree is a basic method for reliability analysis methods, fault analysis and diagnosis ${ }^{[9-10]}$. The classical fault tree analysis uses the AND gate or the OR gate to describe the static failure of the traditional failure ${ }^{[11-12]}$. In the process of fault analysis, the classical fault tree is used to collect and analyze the equipment fault set. In the research of this paper, the fault alarm source of transformer abnormality warning comes from external environment, device body and data transmission body. The data transmission body relies on the dual communication channels of the A manufacturer and the standby B manufacturer. This paper focuses on the correlation and reliability evaluation of the primary and secondary indicators of the device body. The fault tree analysis is shown in Figure 2.

\subsection{Information Representation Based on Fuzzy Comprehensive Evaluation}

In the process of establishing fuzzy comprehensive evaluation system, the appropriate membership function is introduced, and the fuzzy evaluation optimization model is established on this basis. The structural evaluation factors, evaluation criteria and affiliation relationship establishment steps are distinct. According to the affiliation relationship, this paper synthesizes different factor 
information and evaluates the membership level of multi-dimensional information. The steps to construct the model are as follows.

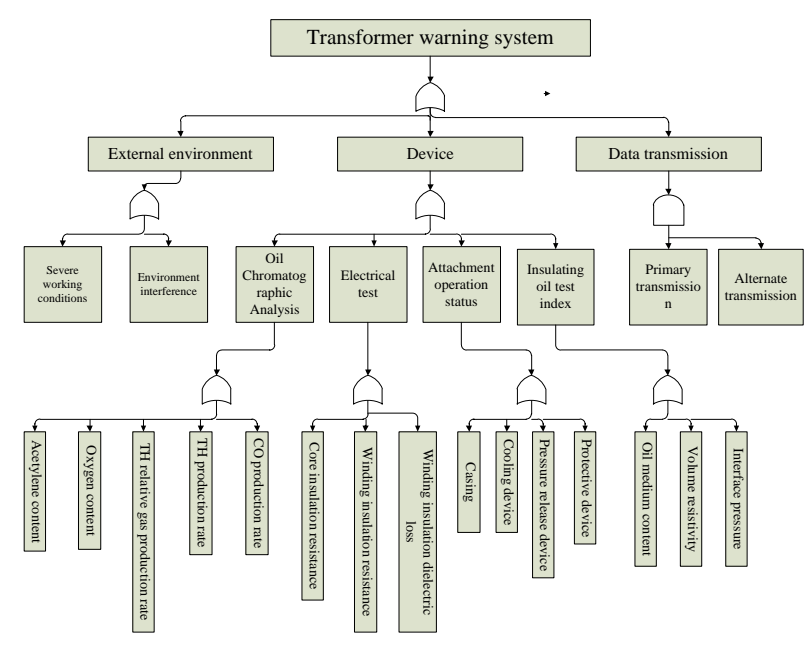

Fig. 2. Fault-Tree of transformer's Abnormal Early-Warning

Step 1: Divide the target status level. According to the operating state of the transformer, this paper is divided into four evaluation levels, namely: normal state, attention state, abnormal state, severe state, recorded as:

$$
V=\left\{v_{1}, v_{2}, v_{3}, v_{4}\right\}
$$

Step 2: Establish a representation set. According to the different attributes of the fault, the $n$ indicators of the evaluation system constitute $n$ representative index sets, recorded as:

$$
U=\left\{\mu_{1}, \mu_{2}, \mu_{3}, \ldots, \mu_{n}\right\}
$$

Step 3: Establish a fuzzy relationship matrix. Firstly, based on the position of the standard indicator $\mu_{i}$ on the membership function continuous function $v_{i}$ on the hierarchical standard set, the membership degree of each evaluation factor to the four evaluation levels is obtained, and the relationship between the two indicators is established. Network establishment consistency matrix to derive fuzzy relation matrix:

$$
R=\left[\begin{array}{cccc}
r_{11} & r_{12} & r_{13} & r_{14} \\
r_{21} & r_{22} & r_{23} & r_{24} \\
\cdots & \cdots & \cdots & \cdots \\
r_{n 1} & r_{n 2} & r_{n 3} & r_{n 4}
\end{array}\right]
$$

Step 4: Determine the prior probability $P=\left\{P_{1}, P_{2}, \cdots P_{n}\right\}$ and $R$ multiplication operation of the four evaluation levels corresponding to each of the $\mathrm{n}$ indicators. The correlation operation between the indicator prior probability and the weight matrix obtains the second-order weight $n$ dimension vector $A$.

$$
A=P \cdot R=\left[s_{1}, s_{2}, s_{3}, s_{4}\right]
$$

In the formula(8), the risk weight set is normalized. In this paper, the prior probabilit $P=\left\{P_{1}, P_{2}, \cdots P_{n}\right\}$ and the fuzzy relation matrix $R$ are combined to obtain the comprehensive membership vector $S$ of each indicator set.

$$
S_{j}=\sum_{i=1}^{n} a_{i j} r_{i j}
$$

In the formula(9), the risk weight value of each indicator is indicated $S_{i}$, and “." is a fuzzy operator, which is defined as multiplication and addition, and $a_{i j}$ is a medium element $A$.

Step 5: Each index is divided by the membership function. There are many types of membership functions. In this paper, the intermediate trapezoidal distribution is selected, and the expression is shown in equation (10).

$$
T(\mathrm{x})=\left\{\begin{array}{lc}
0, & 0 \leq x \leq a \\
(\mathrm{x}-\mathrm{a}) /(\mathrm{b}-\mathrm{a}), & a \leq x \leq b \\
1, & b \leq x \leq c \\
(\mathrm{~d}-\mathrm{x}) /(\mathrm{d}-\mathrm{a}), & c \leq x \leq d \\
0, & x \geq d
\end{array}\right.
$$

Step 6: Finally, the subordinate discriminant obtained by the upper layer is used for the result discriminant formula (11) as follows:

$$
Y=S \times R=\left(\mathrm{s}_{1}, \mathrm{~s}_{2}, \ldots, \mathrm{s}_{n}\right)\left[\begin{array}{cccc}
r_{11} & r_{12} & r_{13} & r_{14} \\
r_{21} & r_{22} & r_{23} & r_{24} \\
\ldots & \ldots & \ldots & \ldots \\
r_{n 1} & r_{n 2} & r_{n 3} & r_{n 4}
\end{array}\right]=
$$

$\left(\mathrm{y}_{1}, \mathrm{y}_{2}, \mathrm{y}_{3}, \mathrm{y}_{4}\right)$

In the formula(11), the comprehensive membership degree vector $S$ obtained by the second indicator layer is matrix-multiplied with the fuzzy evaluation matrix $R$ of the first-level index layer, and the transformer state representation $Y=\left(y_{1}, y_{2}, \cdots y_{n}\right)$ is used to represent the probability of occurrence of each state, and the state is obtained according to the obtained state probability of the representative quantity. Probability becomes the guiding significance of judgment.

\subsection{Classification of health state based on \\ Bayesian discriminant theory}

A typical pattern recognition system consists of two stages: feature extraction and pattern classification. According to Bayesian theory, the posterior probability is defined as:

$$
P\left(\mathrm{G}_{i} \mid \mathrm{x}\right)=p_{i} f_{i}(\mathrm{x}) /\left(\sum_{j=1}^{k} p_{j} f_{j}(\mathrm{x})\right)
$$

In the formula, Bayesian discrimination is based on a population with $p$ dimensions: $\left\{\mathrm{G}_{1}, \mathrm{G}_{2}, \ldots, \mathrm{G}_{k}\right\}$.The probability density function corresponding to each individual is: 
$f_{1}(\mathrm{x}), f_{2}(\mathrm{x}), \ldots, f_{k}(\mathrm{x})$. The prior probability of the population is: $P_{i}(\mathrm{i}=1,2, \ldots, \mathrm{k})$.Gaussian variable in one dimension is: $X \sim N\left(\mu, \sigma^{2}\right)$. The probability density function is given by formula(13):

$$
f_{i}(\mathrm{x})=\exp \left[-(\mathrm{x}-\mu)^{2} / 2 \sigma^{2}\right] / \sqrt{2 \pi \sigma}
$$

In the formula, $\sum$ is covariance matrix of $p \times p$. The multidimensional probability density function is formula (14):

$$
\begin{aligned}
& f_{i}(\mathrm{x})=\exp \left[\left[-(\mathrm{x}-\mu)^{T} \sum^{-1}(\mathrm{x}-\mu)\right] / 2\right] \div \\
& {\left[(2 \pi)^{p / 2}\left|\sum\right|^{1 / 2}\right]}
\end{aligned}
$$

The loss function due to misjudgment is $Z(j \mid i)$, The average loss caused by misjudgment is formula (15):

$$
T(\mathrm{i} \mid \mathrm{x})=\sum_{j \neq i}\left[p_{i} f_{i}(\mathrm{x}) Z(j \mid i) / \sum_{j=1}^{k} q_{j} f_{j}(\mathrm{x})\right]
$$

Multidimensional space is $X=\left(\mathrm{x}_{1}, \mathrm{x}_{2}, \ldots, \mathrm{x}_{p}\right)$, It can be expressed as $X \sim N\left(\mu, \sum\right), \mu$ is a $p$. dimensional vector, (13) expresses the loss when the $i$ population sample is misjudged as the $j$ sample. The discrimination process is simplified to judge only the maximum of $P\left(\mathrm{G}_{i} \mid \mathrm{x}\right)$, this also takes into account the minimum average loss. This is equivalent to judging $p_{i} f_{i}(\mathrm{x})$, its discriminant function:

$$
y_{i}(x)=p_{i} f_{i}(x) ; \mathrm{i}=1,2, \ldots, \mathrm{k}
$$

In the formula, the criterion is: when $x$ falls into $R_{i}$, then $x \in G_{i}$, then $y_{i}(\mathrm{x})=\max _{1 \leq j \leq k} y_{j}(\mathrm{x})$, and $x \in G_{i}$.According to formula (14) and combining formula (16), formula (17) is obtained:

$$
\begin{aligned}
& y_{i}(x)=q \exp \left[-\left(\mathrm{x}-\mu_{i}\right)^{T} \sum_{i}^{-1}\left(\mathrm{x}-\mu_{i}\right) / 2\right] \div \\
& {\left[(2 \pi)^{1 / 2}\left|\sum_{i}\right|^{1 / 2}\right]}
\end{aligned}
$$

The order criterion is:

$$
Z_{i}(\mathrm{x})=\ln \left[(2 \pi)^{-p / 2} \mathrm{y}_{i}(\mathrm{x})\right]
$$

It expands equation (18) into equation (19):

$$
\begin{aligned}
& Z_{i}(x)=\ln p_{i}-\ln \left|\sum_{i}\right| / 2-x^{T} \sum_{i}^{-1} x / 2+ \\
& x^{T} \sum_{i}^{-1} \mu_{i}-\mu_{i}^{T} \sum_{i}^{-1} \mu_{i} / 2 ; \quad i=1,2, \ldots, k
\end{aligned}
$$

When $\sum=\sum_{1}=\sum_{2}=\ldots=\sum_{k}$, Since the second and third terms of equation (19) are not related to $\mathrm{i}$, so equation (19) is simplified to equation (20):

$$
\begin{aligned}
& Z_{i}(x)=\ln p_{i}+x^{T} \sum_{i}^{-1} \mu_{i}-\mu_{i}^{T} \sum_{i}^{-1} \mu_{i} / 2 ; \\
& i=1,2, \ldots, k
\end{aligned}
$$

In the formula, when $\mathrm{k}=2$, if $q_{1}=q_{2}$ and the probability of misjudgment of the two populations is equal, the Bayesian discrimination is equivalent to the distance criterion.

\section{COLLABORATIVE DISCRIMINATION METHOD}

Based on the method of fuzzy comprehensive evaluation and Bayesian discrimination, a power transformer state discrimination and early warning model was established. The judgment process of this model is shown in Figure 3.

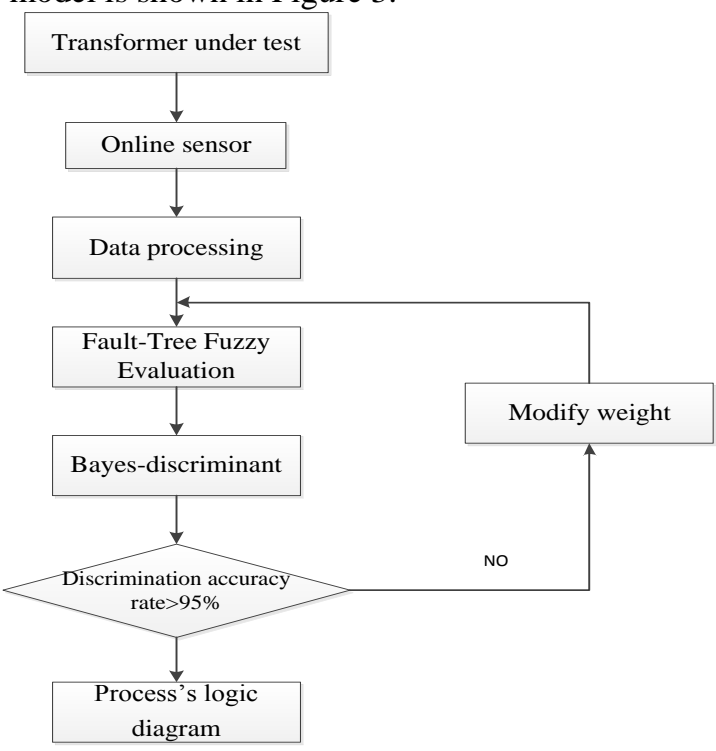

Fig. 3. BM Judgment

The specific evaluation steps of BM Judgment are as follows:

1) Monitor the sample to be tested to obtain the measured values of various indicators of the power transformer.

2) The measurement results are initialized and quantified according to the state evaluation system.

3) The evaluation result of the sample set is obtained based on the fuzzy comprehensive evaluation model of the transformer.

4) Based on the evaluation results of the fuzzy comprehensive evaluation model, the Bayesian discriminant model is used to calculate the correct rate of sample discrimination. If the correct rate is higher than $95 \%$, then the model is output. If the correct rate is lower than $95 \%$, return to step 2 and update Index weight, improve model evaluation model.

5) According to the diagnosis results, determine the operating status of the power transformer and propose early warning recommendations.

6) Go to the next system according to the result from step 5 .

The classical fuzzy evaluation method and Bayesian discriminant method are used to verify the results, and the prediction results are compared with the historical data to make the correct rate reach more correct, and then the evaluation system is established. The judgment process of the 
collaborative evaluation system is shown in Figure 4 and can be divided into the following stages.

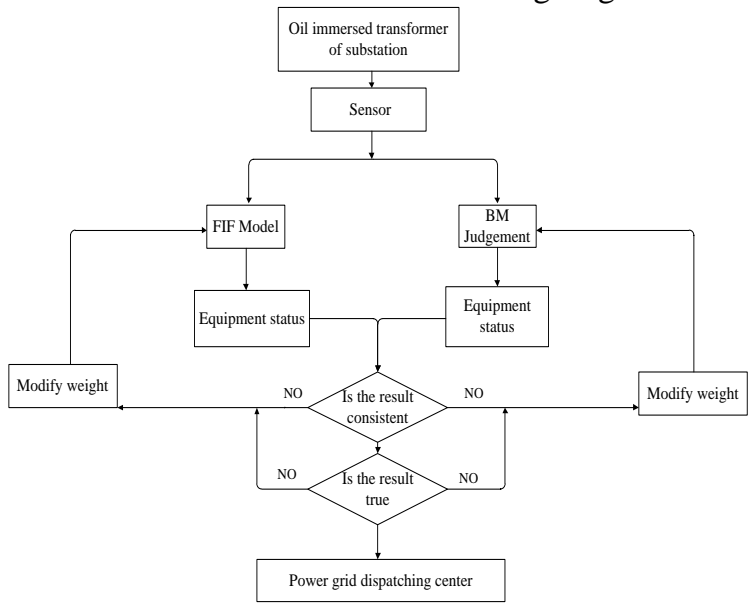

Fig. 4. Process's logic diagram

Operation steps of process's logic diagram:

1) Establish the monitored transformer voltage level. Different levels of transformer related parameters have different measurement standards.

2) Collect various types of information obtained by the sensor, and use the fuzzy evaluation method and Bayesian discrimination method to discriminate the state of the transformer.

3) If the result is the same, go to step 4, otherwise go to step 5 .

4) According to the real data, if the result is the same, go to step 6, otherwise go to step 5 .

5) Perform weight modification on the fuzzy evaluation method and modify the coefficient of the Bayesian discriminant formula.

6) New sample can be used to conduct power transformer state assessment through the collaborative evaluation system, the evaluation result can be used to assist the power grid operation and maintenance personnel to handle.

\section{CASE ANALYSIS}

In this paper, the father and sub-index test data of 35 oil-immersed transformers from 10 substations are used to construct system. The transformer state is composed of normal, attention, abnormal and severe states. The first-level index consists of oil chromatographic analysis, electrical test, accessory operation and insulating oil test indicators. It is calculated by the method in Section 1.1 to establish the weights and probability of occurrence of each abnormality. The two intermediate quantities are multiplied to obtain ndimensional weights vector.

First of all, the correct rate of judgment results needs to be improved. This paper needs to compare the results of fuzzy evaluation methods and Bayesian discrimination. Secondly, after the system is built, it is necessary to systematically discriminate the test set, and compare the judgment result of the collaborative method with the historical data to judge the correctness of the experiment.

Taking 16 sample sets to calculate the secondary indicators value and the evaluation values of the first-level indicators. At this time, according to the two-layer fuzzy comprehensive evaluation method ${ }^{[13-14]}$, the weights of the four first-level indicators are allocated as $\{0.25,0.25$. $0.25,0.25\}$, the results are weighted and summed, $z$ as a comprehensive score, and the results are shown in Table 7.

Table 7. Fuzzy evaluational results of father index

\begin{tabular}{ccccccc}
\hline Group & $\mu_{1}$ & $\mu_{2}$ & $\mu_{3}$ & $\mu_{4}$ & $z$ & category \\
\hline 1 & 0.21 & 0.15 & 0.21 & 0.15 & 0.18 & 1 \\
2 & 0.15 & 0.11 & 0.20 & 0.15 & 0.1525 & 1 \\
3 & 0.35 & 0.10 & 0.08 & 0.20 & 0.1825 & 1 \\
4 & 0.10 & 0.11 & 0.21 & 0.05 & 0.1175 & 1 \\
5 & 0.18 & 0.21 & 0.05 & 0.17 & 0.1525 & 1 \\
6 & 0.38 & 0.49 & 0.32 & 0.21 & 0.35 & 2 \\
7 & 0.35 & 0.42 & 0.39 & 0.25 & 0.3525 & 2 \\
8 & 0.51 & 0.21 & 0.43 & 0.48 & 0.4075 & 2 \\
9 & 0.52 & 0.28 & 0.47 & 0.52 & 0.4475 & 2 \\
10 & 0.73 & 0.75 & 0.89 & 0.95 & 0.83 & 4 \\
11 & 0.54 & 0.62 & 0.65 & 0.73 & 0.635 & 3 \\
12 & 0.56 & 0.73 & 0.63 & 0.74 & 0.665 & 3 \\
13 & 0.73 & 0.80 & 0.69 & 0.73 & 0.735 & 3 \\
14 & 0.72 & 0.85 & 0.87 & 0.85 & 0.8225 & 4 \\
15 & 0.83 & 0.76 & 0.70 & 0.90 & 0.7975 & 4 \\
16 & 0.94 & 0.70 & 0.82 & 0.73 & 0.7975 & 4 \\
\hline
\end{tabular}

The comprehensive evaluation process of the secondary indicators will not be described in detail. Table 7 gives the primary indicator status values. After weighting the primary indicator status values, the resulting status values represent four different states: normal (1), attention (2), abnormal (3), severe (4). The severe state is equally divided into 4 data fields, and the data field is between 0 and 1 . In the research process of this paper, the secondary indicator status value is used as the initial data of Bayesian discriminant, and the initial data set is corresponding to the transformer status value. The data is trained to obtain the discriminant criterion, and the discriminant essence is used to check the health status of the device. According to the training of 16 sets of data sets, the generated Bayesian discriminant category distribution is shown in Figure 5. 


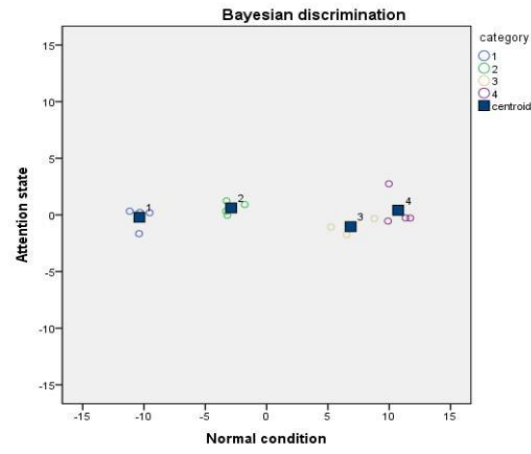

Fig. 5. Bayes-discriminant result

As shown in FIG 5, 16 sets of data are distributed around the four groups of centroids in four states, and the group centroid represents the standard score of the four state quantities under computer simulation. In the figure, between the abnormal state and the severe state, the point are in a fuzzy state, and according to the point, the state is closer to the abnormal state, so it is classified into third categories.

The computer simulation calculation is based on the covariance construction of the 16 sets of data sets in Table 7 according to Section 2.3. The Bayesian discriminant function thus establishes the correlation coefficient. The corresponding coefficients of the four discriminant functions are shown in Table 8.

Table 8. Coefficient of Classificational Function

\begin{tabular}{ccccc} 
& \multicolumn{4}{c}{ category } \\
\cline { 2 - 5 } & 1 & 2 & 3 & 4 \\
\hline$\mu_{1}$ & 35.100 & 82.066 & 123.007 & 154.731 \\
$\mu_{2}$ & 57.039 & 144.270 & 270.663 & 308.657 \\
$\mu_{3}$ & 48.992 & 126.986 & 208.772 & 255.639 \\
$\mu_{4}$ & 22.335 & 58.065 & 126.969 & 140.666 \\
(constant) & -14.022 & -80.841 & -250.781 & -346.849
\end{tabular}

According to the classification function coefficient table of Table 7, the class function is constructed. The four formulas correspond to the normal, attention, abnormal, and severe states respectively. The larger value of the four calculation result will be determined to be the state. The discriminant function is as shown in formula (21).

The experimental calculation process also needs to verify its scientificity. The 16 data are not characterized by cross-border indicators. When the data is completely out of the specified range, this article will eliminate or correct them.

$$
\begin{aligned}
& f_{1}=35.100 \mu_{1}+57.039 \mu_{2}+48.992 \mu_{3} \\
& +22.335 \mu_{4}-14.022 ; \\
& f_{2}=82.066 \mu_{1}+144.270 \mu_{2}+126.986 \mu_{3} \\
& +58.065 \mu_{4}-80.841 ; \\
& f_{3}=123.007 \mu_{1}+270.663 \mu_{2}+208.772 \mu_{3} \\
& +126.969 \mu_{4}-250.781 ; \\
& f_{4}=154.731 \mu_{1}+308.657 \mu_{2}+255.639 \mu_{3} \\
& +140.666 \mu_{4}-346.849 ;
\end{aligned}
$$

The four discriminant formulas listed in Equation 10, and the risk assessment values of the four first-level indicators are used as variables. The state value depends on the size of the function. Therefore, the rigor of the function is tested. This paper identifies the data of the nine transformers tested, and the set is shown in Table 9:

Table 9. Index Weight Table for Testing Transformers

\begin{tabular}{ccccc} 
Group & $\mu_{1}$ & $\mu_{2}$ & $\mu_{3}$ & $\mu_{4}$ \\
\hline 17 & 0.21 & 0.15 & 0.21 & 0.15 \\
18 & 0.15 & 0.11 & 0.20 & 0.15 \\
19 & 0.35 & 0.10 & 0.08 & 0.20 \\
20 & 0.10 & 0.11 & 0.21 & 0.05 \\
21 & 0.18 & 0.21 & 0.05 & 0.17 \\
22 & 0.38 & 0.49 & 0.32 & 0.21 \\
23 & 0.35 & 0.42 & 0.39 & 0.25 \\
24 & 0.51 & 0.21 & 0.43 & 0.48 \\
25 & 0.52 & 0.28 & 0.47 & 0.52 \\
\hline
\end{tabular}

Comparison of fuzzy evaluation results and Bayesian discrimination results based on with after training 16 transformer data. Then, we test the data of 9 unidentified transformers. The results are shown in Table 10.

Table 10. Comparison of Parallel Methods

\begin{tabular}{cccccc} 
Group & FTF & BM & Group & FTF & BM \\
\hline 1 & 1 & 1 & 14 & 4 & 4 \\
2 & 1 & 1 & 15 & 4 & 4 \\
3 & 1 & 1 & 16 & 4 & 4 \\
4 & 1 & 1 & 17 & 1 & 1 \\
5 & 1 & 1 & 18 & 1 & 1 \\
6 & 2 & 2 & 19 & 3 & $\# 2$ \\
7 & 2 & 2 & 20 & 3 & 3 \\
8 & 2 & 2 & 21 & 3 & 3 \\
9 & 2 & 2 & 22 & 4 & 4 \\
10 & 4 & 4 & 23 & 4 & 4 \\
11 & 3 & 3 & 24 & 3 & 3 \\
12 & 3 & 3 & 25 & 2 & 2 \\
13 & 3 & 3 & & & \\
\hline
\end{tabular}


It can be seen from the conclusion data of Table 10 , the results of the first 16 groups using the two methods are one-to-one correspondence. The first to fifth groups are all in a normal state, and the sixth to the ninth groups are all in a state of attention, The 11th to the 13th groups are all abnormal, and the 10th group and the 14th to 16th groups are all in a serious state. In the test group, the results of the other eight groups are the same except for the conclusions of the data of the 19th group. the conclusions of the 25 groups are basically the same, and the 19th group is the abnormal state. The reason may be that the discriminant point is at the Bayesian discriminant boundary, and the discriminant error can be reduced if the correction coefficient is corrected and more historical data is absorbed.

In this example, the fault identification and diagnosis and early warning of power transformers are carried out. Based on the results of the twomethod test, a state discriminant model based on Bayesian discriminant and fuzzy evaluation system is established ${ }^{[15]}$.

After detailed comparison and comparison of the two collaborative methods in the system, the paper manually revised the system and perfected the system structure. According to the correction of the 9 groups of data in the test process, this paper systematically discriminates the undetected all groups of data. This experiment tests all above data,these data include training data and untested data. 1 16 groups are training data, and 26 35 groups are untested data. Such cross-experiments make the test results more convincing. The result are as shown in Table 11.

The system is constructed by using initial 16 sets of data, and it's parameter are modified by using 9 test data. Finally, we used initial 16 sets of data and another 10 sets of test data to determine the health status of the transformer, and the results met expectations. It can be seen from the results that the accuracy of the judgment using this method is very high, but at the same time, we also found that the data of group 26 is not consistent with the actual. This error is related to the second modification of the parameters, but a large amount of data Training can change this situation, and we will further study this.

As shown in FIG 6,the experiment showed the results of 26 sets of data in a two-dimensional plan. Red, green, gray, and purple represent four different working states, and yellow does not belong to any one state. Such errors are essentially due to insufficient data in the sample, which in turn The boundaries cannot be clear, but we may be able to solve this error by training with multiple data.

\begin{tabular}{ccccccc}
\multicolumn{6}{c}{ Table 11. Discrimination of test group } \\
\hline & $\mu_{1}$ & $\mu_{2}$ & $\mu_{3}$ & $\mu_{4}$ & Actual & System \\
Group & & & & & category & category \\
\hline 1 & 0.21 & 0.15 & 0.21 & 0.15 & 1 & 1 \\
2 & 0.15 & 0.11 & 0.20 & 0.15 & 1 & 1 \\
3 & 0.35 & 0.10 & 0.08 & 0.20 & 1 & 1 \\
4 & 0.10 & 0.11 & 0.21 & 0.05 & 1 & 1 \\
5 & 0.18 & 0.21 & 0.05 & 0.17 & 1 & 1 \\
6 & 0.38 & 0.49 & 0.32 & 0.21 & 2 & 2 \\
7 & 0.35 & 0.42 & 0.39 & 0.25 & 2 & 2 \\
8 & 0.51 & 0.21 & 0.43 & 0.48 & 2 & 2 \\
9 & 0.52 & 0.28 & 0.47 & 0.52 & 2 & 2 \\
10 & 0.73 & 0.75 & 0.89 & 0.95 & 4 & 4 \\
11 & 0.54 & 0.62 & 0.65 & 0.73 & 3 & 3 \\
12 & 0.56 & 0.73 & 0.63 & 0.74 & 3 & 3 \\
13 & 0.73 & 0.80 & 0.69 & 0.73 & 3 & 3 \\
14 & 0.72 & 0.85 & 0.87 & 0.85 & 4 & 4 \\
15 & 0.83 & 0.76 & 0.70 & 0.90 & 4 & 4 \\
16 & 0.94 & 0.70 & 0.82 & 0.73 & 4 & 4 \\
26 & 0.22 & 0.14 & 0.25 & 0.13 & 1 & 1 \\
27 & 0.13 & 0.14 & 0.23 & 0.12 & 1 & 1 \\
28 & 0.26 & 0.11 & 0.06 & 0.19 & 1 & 1 \\
29 & 0.28 & 0.26 & 0.53 & 0.42 & 2 & 2 \\
30 & 0.53 & 0.48 & 0.37 & 0.46 & 2 & 2 \\
31 & 0.67 & 0.72 & 0.74 & 0.81 & 3 & 3 \\
32 & 0.35 & 0.78 & 0.36 & 0.65 & 3 & $\# 2$ \\
33 & 0.51 & 0.31 & 0.43 & 0.88 & 3 & 3 \\
34 & 0.78 & 0.79 & 0.87 & 0.92 & 4 & 4 \\
35 & 0.84 & 0.86 & 0.90 & 0.96 & 4 & 4 \\
\hline & & & & & &
\end{tabular}

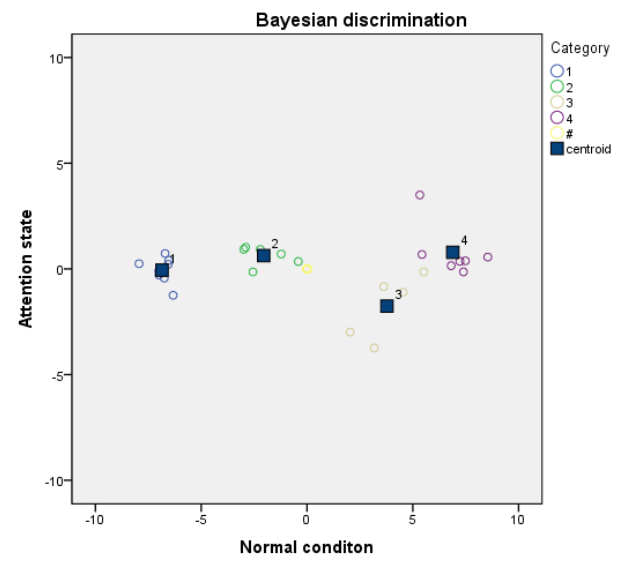

Fig. 6. Second Bayes-discriminant result

\section{CONCLUSIONS}

As a classifier, Bayesian networks have expanded with various structures and research methods. Discriminant learning is an extended learning method for Bayesian network classifiers. Discriminant Bayesian network classifiers have been studied in recent years.

China State Grid Corporation of China monitors various devices online through many methods, including the fuzzy evaluation system in this paper, but this paper also adds the Bayesian discrimination system. The simulation experiment based on the combination of two discrimination methods is the 
fifth section in this article, which has verified the correctness of this theory.

The correct rate determined by the experiment is in line with expectations. The transmission and distribution network can use the fuzzy evaluation method and the Bayesian discrimination method to jointly develop a health condition assessment method for multiple power equipments. Based on the fuzzy evaluation method, the Bayes discriminant function is constructed by using the state values of the four first-level indicators. In summary,this research method has several advantages:

(1) This paper compares the fuzzy evaluation system and the Bayesian discriminant system:

The existing fuzzy evaluation and Bayesian discriminant learning algorithms are elaborate, and then the experimental results of the two discriminants are compared.

(2) A faster and more accurate learning algorithm is proposed:

The scope of application of this method can also be applied to equipment health assessment in different engineering fields. The innovation of computer also gives it new vitality, reducing decision time and getting higher work efficiency.

(3) Condition evaluation of electrical equipment based on Bayesian discrimination:

The construction of the system not only relies on artificial weights and expert opinions. This paper combines Bayesian discrimination to reduce the interference of artificial factors, and the result is expressed by the combination of artificial weight and machine discrimination.

\section{SOURCE OF FUNDING}

This work is supported by National Natural Science Foundation of China (Grant, No.61673093).

\section{REFERENCES}

1. Yu H, Wu ZR, Bao TF, Zhang L. Multivariate analysis in dam monitoring data with PCA. Science China Technological Sciences. 2010; 53(4): 10881097. https://doi.org/10.1007/s11431-010-0060-1

2. Hao M, Sun X, Yin L and Ding Q. Prediction of GAMIT baseline solution based on Bayesian classifier. 2018 13th IEEE Conference on Industrial Electronics and Applications (ICIEA). 2018; pp. 2735-2739. https://doi.org/10.1109/ICIEA.2018.8398174

3. Chiacchio F, D'Urso D, Compagno L, Pennisi M, Pappalardo F, Manno G. A stochastic hybrid fault tree automaton for the modelling and simulation of dynamic reliability problems. Expert Systems with Applications. 2016;47(3):42-57. https://doi.org/10.1016/j.eswa.2015.10.046

4. Li ZJ, Chi GT. Factors study of credit risks of farmer loans based on projection pursuit. Proceedings of 2013 IEEE International Conference on Service Operations and Logistics, and Informatics. 2013: 274-277. https://doi.org/10.1109/SOLI.2013.6611424
5. Jiang SC, Chin KS, Wang L, Qu G, K.L. T. Modified genetic algorithm - based feature selection combined with pre-trained deep neural network for demand forecasting in outpatient department. Expert Systems With Applications. 2017; 82(3): 1726-1730. https://doi.org/10.1016/j.eswa.2017.04.017

6. Li JZ, Zhang QG, Wang K, Wang JY, Zhou TC, Zhang YY. Optimal dissolved gas ratios selected by genetic algorithm for power transformer fault diagnosis based on support vector machine. IEEE Transactions on Dielectrics and Electrical Insulation. 2016;23(2):1198-1206.

https://doi.org/10.1109/TDEI.2015.005277

7. Huang YC, Sun HC. Dissolved gas analysis of mineral oil for power transformer fault diagnosis using fuzzy logic. IEEE Transactions on Dielectrics and Electrical Insulation. 2013; 20(3): 974-981. https://doi.org/10.1109/TDEI.2013.6518967

8. Dai JJ, Song H, Sheng GH, Jiang XC. Dissolved gas analysis of insulating oil for power transformer fault diagnosis with deep belief network. IEEE Transactions on Dielectrics and Electrical Insulation. 2017;24(5):2828-2835. https://doi.org/10.1109/TDEI.2017.006727

9. Chen LL, Yu Z, Ye PF, Zhang J, Zou JZ. Detecting driving stress in physiological signals based on multimodal feature analysis and kernel classifiers. Expert Systems with Applications. 2017; 76(3): 1987-1993. https://doi.org/10.1109/TDEI.2017.006727

10. Purba JH. A fuzzy-based reliability approach to evaluate basic events of fault tree analysis for nuclear power plant probabilistic safety assessment. Annals of Nuclear Energy. 2014; 70(3): 21-29. https://doi.org/10.1016/j.anucene.2014.02.022

11. Ruijters E, Stoeling M. Fault tree analysis: A survey of the state-of-the-art in modeling, analysis and tools. Computer Science Review. 2015; 15-16(3): 29-62. https://doi.org/10.1016/j.cosrev.2015.03.001

12. Biswas SS, Srivastava AK, Whitehead D. A RealTime Data-Driven Algorithm for Health Diagnosis and Prognosis of a Circuit Breaker Trip Assembly. IEEE Transactions on Industrial Electronics. 2015; 62(6):3822-3831. https://doi.org/10.1109/TIE.2014.2362498

13. Duval M. A review of faults detectable by gas-in-oil analysis in transformers. IEEE Electrical Insulation Magazine.2002;18(3):8-17. https://doi.org/10.1109/MEI.2002.1014963

14. Dai J, Song H, Sheng G, Jiang X. Dissolved gas analysis of insulating oil for power transformer fault diagnosis with deep belief network. IEEE Transactions on Dielectrics and Electrical Insulation. 2017;24(5)2828-2835 https://doi.org/10.1109/TDEI.2017.006727

15. He X, Ai Q, Qiu RC, Huang W, Piao L, Liu H. A Big Data Architecture Design for Smart Grids Based on Random Matrix Theory. IEEE Transactions on Smart Grid.2017;8(2):674-686. https://doi.org/10.1109/TSG.2015.2445828

Received 2019-10-16

Accepted 2020-03-03

Available online 2020-04-28 


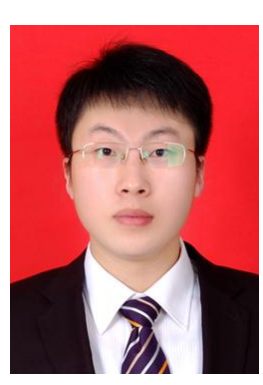

Dianyang LI, doctor, Senior engineer, Research on power grid operation and power dispatch control technology and application.

E-mail: 2841385779@qq.com;

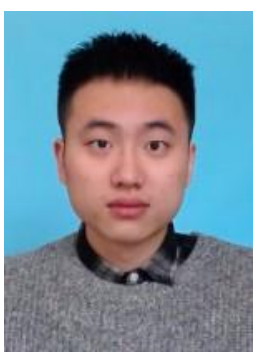

Shanyuan WANG, Postgraduate, Research on Smart grid and fault diagnosis.

E-mail: wsyneu@163.com; 